\title{
The New Small Wheel upgrade project of the ATLAS Experiment
}

\author{
Valerio D’Amico ${ }^{a, b, 1, *}$ \\ ${ }^{a}$ Dipartimento di Matematica e Fisica, Università degli Studi Roma Tre, \\ Via della Vasca Navale 84, Roma, Italia \\ ${ }^{b}$ INFN sezione di Roma Tre, \\ Via della Vasca Navale 84, Roma, Italia \\ E-mail: valerio.damico@cern.ch, valerio.damico@uniroma3.it, \\ valerio.damico@roma3.infn.it
}

The ATLAS experiment is currently upgrading the first muon station in the high-rapidity region with the construction of new detector structures, named New Small Wheels (NSW), based on large-size multi-gap resistive strips Micromegas technology and small-strip Thin Gap Chambers (sTGC). The NSW system will be installed in the ATLAS underground cavern during the LHC long shutdown 2 to enter in operation for Run3. The construction of Micromegas and sTGC detectors is completed at the construction sites distributed all around the world. At CERN, the final validation and integration of the modules in sectors composing the wheel is well advanced, as well as the installation on the final structure and the commissioning on surface. In this presentation the motivation of the NSW upgrade and the current status of the project will be reviewed, with particular focus on the main challenges and the measured performance of the system.

\footnotetext{
*** The European Physical Society Conference on High Energy Physics (EPS-HEP2021), ***

*** 26-30 July $2021 * * *$

*** Online conference, jointly organized by Universität Hamburg and the research center DESY ***
}

${ }^{1}$ on behalf of the ATLAS Muon Collaboration

${ }^{*}$ Speaker 


\section{Introduction}

The instantaneous luminosity provided by the Large Hadron Collider (LHC) during the Run3 and the High Luminosity LHC (HL-LHC) phase is planned to increase up to $7.5 \times 10^{34} \mathrm{~s}^{-1} \mathrm{~cm}^{-2}$. A huge increase of particle rates is expected, mainly in the high-rapidity regions of the ATLAS experiment [1]. This would result in a loss in efficiency of the current muon detectors of the Small Wheel, the first end-cap station of the Muon Spectrometer. The larger amount of particles would affect the trigger and tracking performances, introducing the triggering of many fake non-prompt muons and increasing the background rates. The trigger rate would exceed the readout bandwidth dedicated to the ATLAS L1 muon trigger and the thresholds should be increased, losing low- $p_{\mathrm{T}}$ muons at L1 trigger level. For these reasons, an upgrade of the the Muon Spectrometer has been designed to cope with the expected flux of particles in the forward regions $1.3<|\eta|<2.7$, maintaining the design trigger and tracking performances for the entire Run3 and HL-LHC: the New Small Wheel [2]. Two new detector technologies, Micromegas (MM) [3] and small-strip Thin Gap Chambers (sTGC) [4], are exploited to provide excellent tracking and trigger performances in this new demanding conditions. The main requirements are to substantially reduce the fakes trigger rate at $\mathrm{L} 1$, to reconstruct the online muon tracks with $95 \%$ efficiency, and to provide excellent spatial and angular resolutions: less $50 \mu \mathrm{m}$ for the reconstructed segments in the wheel, and less than $1 \mathrm{mrad}$ for online matching with the Big Wheel. Each wheel is made of 16 sectors and each sector is composed of two sTGC wedges and two MM wedges as shown in Figure 1. The sTGC wedges are made of three quadruplets modules, each composed of four sTGC layers. The MM wedges are, instead, made of two quadruplet modules, made by 2 precision and 2 stereo strip layers, with the readout strips tilted by respectively $+1.5^{\circ}$ and $-1.5^{\circ}$ providing second coordinate information. Two MM wedges are assembled to compose a double-wedge. NSW sectors are assembled at CERN starting from a MM double-wedge: on each side of the double-wedge is mounted an sTGC wedge to form a sector with eight sTGC and eight MM detector layers, as visible from Figure 1.

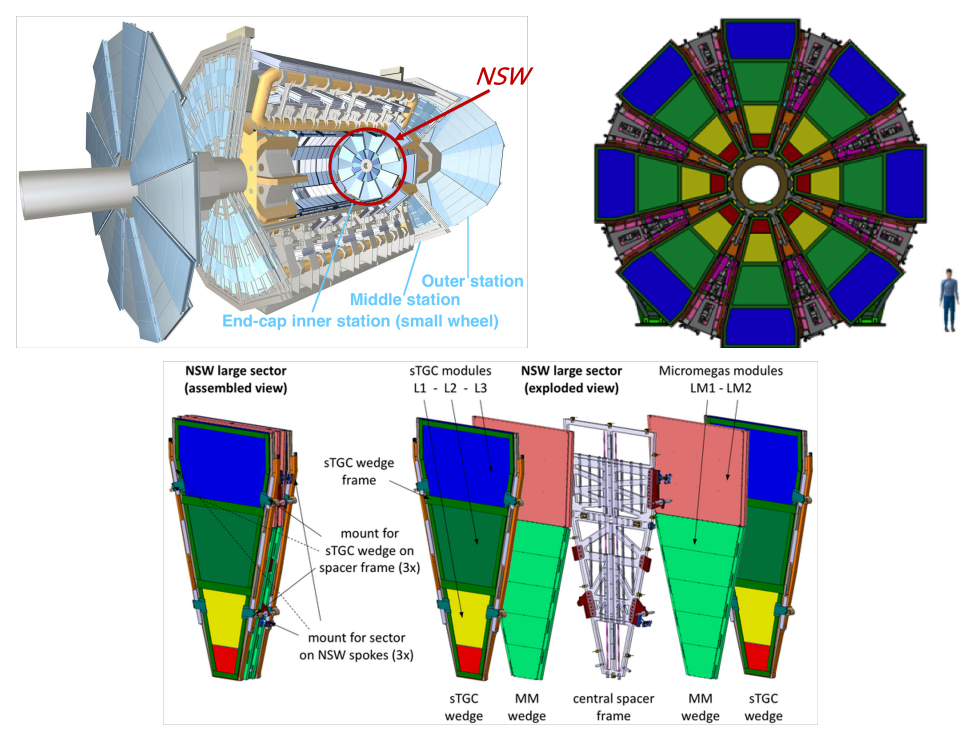

Figure 1: Schematic diagram of the NSW inside the ATLAS detector, showing the structure of a sector of the NSW and the position of MM and sTGC chambers. 


\section{Micromegas}

The Micromegas detectors, whose schematic description is shown in Figure 2, consist in $5 \mathrm{~mm}$ drift gap separated from an amplification gap of $128 \mu \mathrm{m}$ by a very thin metallic mesh, having a wire diameter of $30 \mu \mathrm{m}$ with an opening of $70 \mu \mathrm{m}$. The metallic mesh is floating over pillars of insulating material placed on the anode readout and it is transparent to electrons, providing also a fast evacuation of the positive ions. Both regions are filled with a gas mixture of $\mathrm{Ar}: \mathrm{CO}_{2}$ (93\%:7\%). The readout layer is composed of resistive strips of $300 \mu \mathrm{m}$ width with a pitch of 450 $\mu \mathrm{m}$ capacitively coupled with readout $\mathrm{Cu}$ strips placed below them. The resistivity of $\sim 10 \mathrm{M} \Omega$ of the resistive layer protects the $\mathrm{Cu}$ strips from the sparks that may occur in the amplification gap.

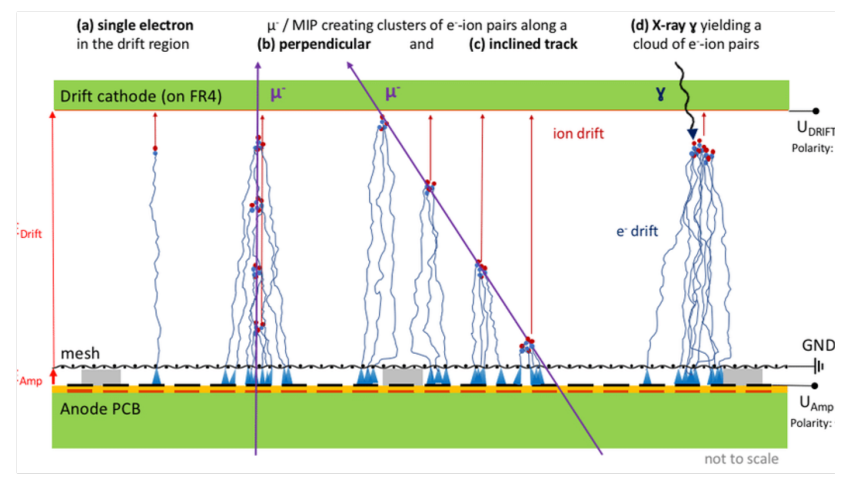

Figure 2: Sketch of the layout cross-section and operating principle of a MM detector.

The MM quadruplet assembly is performed in different construction sites in Italy, Germany, France, Greece and Russia. Micromegas double-wedge assembly and validation, checking the high voltage (HV) stability, the gas leak, the electronic noise and the connectors, is done at CERN. Each doublewedge is tested at a cosmic ray stand at CERN to evaluate the reconstruction efficiency and solve potential electronics or $\mathrm{HV}$ issues. This detector is primarily used for tracking, having a spatial resolution $<100 \mu \mathrm{m}$ for small angles and $72 \mu \mathrm{m}$ for perpendicular tracks as seen from test beam data on the first production modules, reconstructing the position with the centroid method. Results from cosmic stand and test beam data are shown in Figure 3.

\section{Small-strip Thin Gap Chambers}

The sTGC are multiwire ionization chambers operated in quasi-saturated mode. Each layer is made of two cathodes with anode wires in the gap between them, as visible from Figure 4. One cathode is divided into large pads used for the trigger, with a requirement of a 3-out-of-4 coincidence within a quadruplet. The other cathode is made of strips with a $3.2 \mathrm{~mm}$ pitch, providing precision track reconstruction in the $\eta$ direction. Wires have a $1.5 \mathrm{~mm}$ pitch in the direction orthogonal to the strips and can provide second coordinate position information. The sTGC are operated at a voltage of $2.9 \mathrm{kV}$ with a 55:45 gas mixture of $\mathrm{CO}_{2}$ and n-pentane. At this working point they provide $95 \%$ reconstruction efficiency and $75 \mu \mathrm{m}$ position resolution for perpendicular tracks, see Figure 4, with the position obtained from a Gaussian fit of the strip pulse height distribution within a cluster. sTGC quadruplets are produced in Canada, Chile, China, Israel, Russia and then shipped to CERN for wedge assembly and testing. Several tests are done to check potential problems with the modules, 

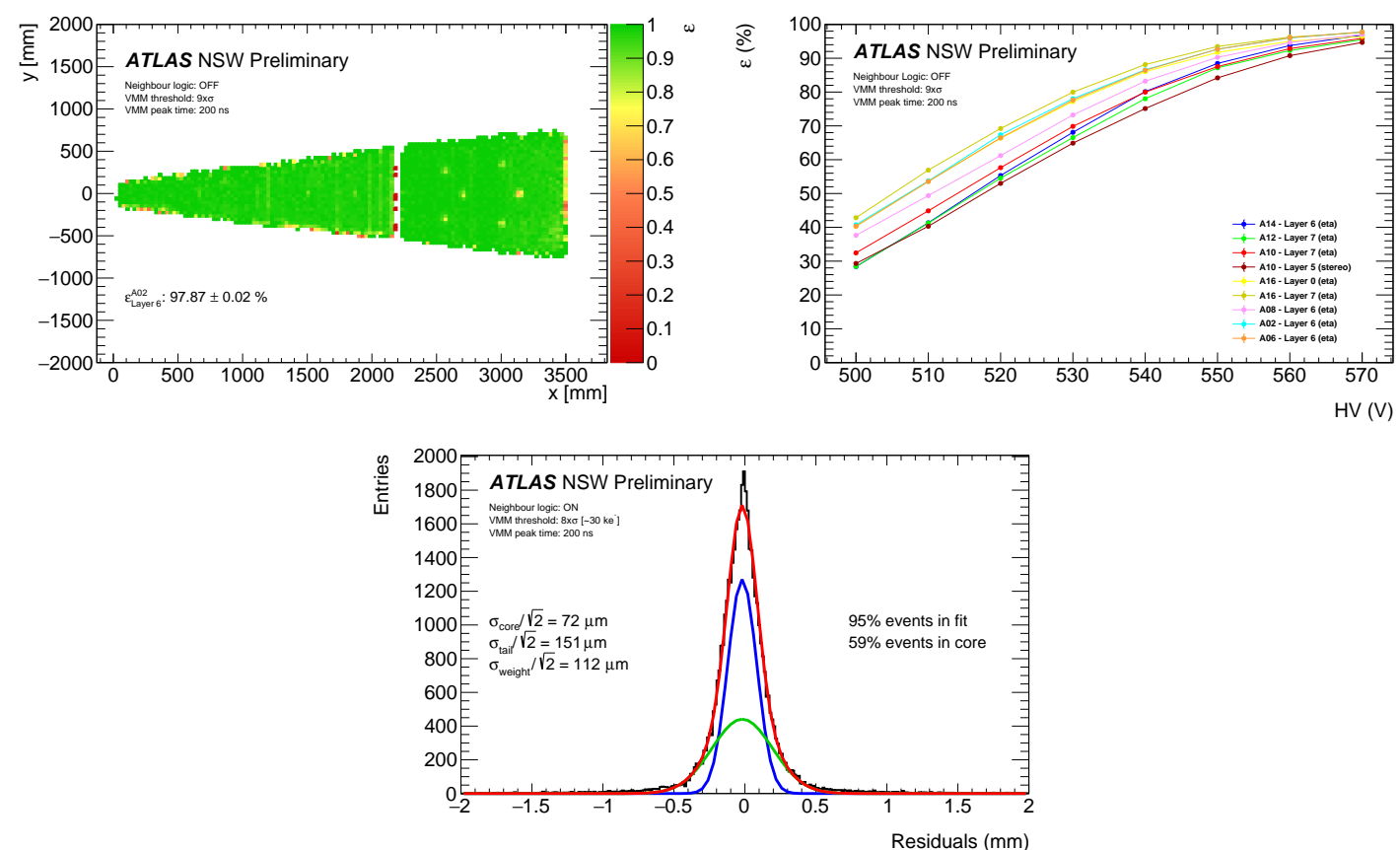

Figure 3: Efficiency of a layer of a MM double-wedge evaluated from cosmic data (left). Efficiency curve of MM double-wedges evaluated with cosmics at different voltages of the readout plane (center). Position resolution of a MM chamber evaluated with test-beam data (right) [5].
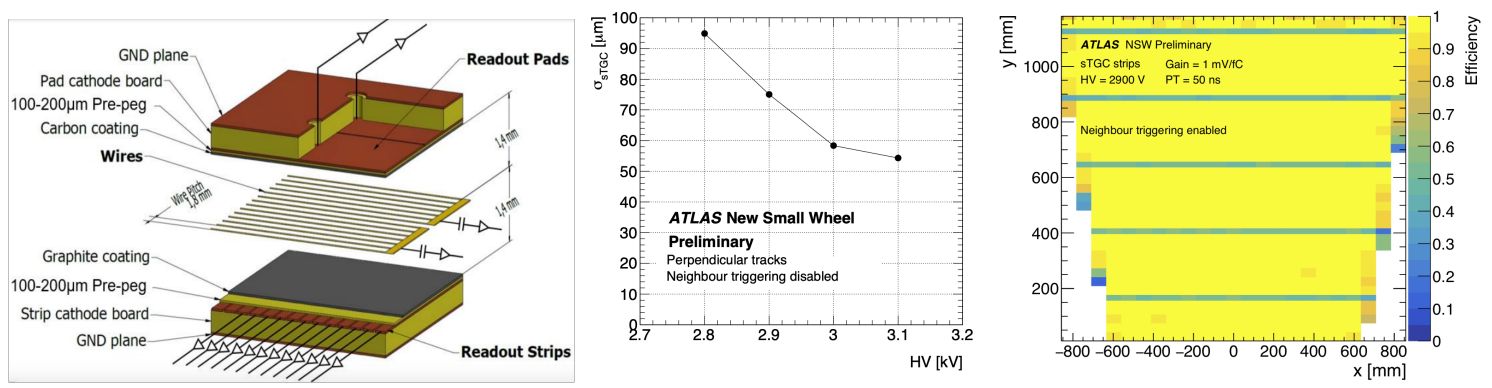

Figure 4: Picture of the layout of an sTGC detector (left). Position resolution of a sTGC detector evaluated with test-beam data at different HV working points (center). Efficiency of a layer of a sTGC module evaluated from cosmic data (right) [5].

among which gas leak, electrode connectivity, low voltage and high voltage stability tests. Then, the strip misalignment is measured with X-rays on each sTGC assembled wedge.

\section{Status of the project and conclusions}

The sTGC and MM production is complete and 32 out of 32 sectors required for the two NSWs have been assembled. The mechanical assembly and integration of all the 32 NSWs sectors has been completed. The first wheel has completed the installation of the services and has been validated. The transport to the experimental cavern and the placement in its position inside the ATLAS detector 
were completed in July. The second wheel is completed and ready to be moved to the cavern to complete the detector, to start the commissioning phase and to take data during the Run3.

\section{References}

[1] ATLAS Collaboration, 2008 JINST 3 S08003.

[2] ATLAS Collaboration, New Small Wheel Technical Design Report, CERN-LHCC-2013-006.

[3] Y. Giomataris et al., MICROMEGAS: A High granularity position sensitive gaseous detector for high particle flux environments, Nucl. Instrum. Meth. A 376 (1996) 29.

[4] S. Majewski et al., A thin multiwire chamber operating in the high multiplication mode, Nucl. Instrum. Meth. 217 (1983) 265.

[5] ATLAS Collaboration, New Small Wheel Public Results, ATLAS public repository. 